\title{
Exchange and correlation effects on plasmon dispersions and Coulomb drag in low-density electron bilayers
}

\author{
S. M. Badalyan, $, 2, *$ C. S. Kim, ${ }^{2}$ G. Vignale, ${ }^{3}$ and G. Senatore ${ }^{4,5}$ \\ ${ }^{1}$ Radiophysics Department, Yerevan State University, 1 A. Manoukian Street, Yerevan, 375025 Armenia \\ ${ }^{2}$ Department of Physics and Institute for Condensed Matter Theory, Chonnam National University, Gwangju 500-757, Korea \\ ${ }^{3}$ Department of Physics and Astronomy, University of Missouri-Columbia, Columbia, Missouri 65211, USA \\ ${ }^{4}$ Dipartimento di Fisica Teorica, Università di Trieste, Strada Costiera 11, 34014 Trieste, Italy \\ ${ }^{5}$ INFM-CNR Democritos National Simulation Center, Trieste, Italy
}

(Received 11 May 2005; revised manuscript received 2 November 2006; published 23 March 2007)

\begin{abstract}
We investigate the effect of exchange and correlation (XC) on the plasmon spectrum and the Coulomb drag between spatially separated low-density two-dimensional electron layers. We adopt a different approach, which employs dynamic XC kernels in the calculation of the bilayer plasmon spectra and of the plasmon-mediated drag, and static many-body local field factors in the calculation of the particle-hole contribution to the drag. The spectrum of bilayer plasmons and the drag resistivity are calculated in a broad range of temperatures taking into account both intra- and interlayer correlation effects. We observe that both plasmon modes are strongly affected by XC corrections. After the inclusion of the complex dynamic XC kernels, a decrease of the electron density induces shifts of the plasmon branches in opposite directions. This is in stark contrast with the tendency observed within random phase approximation that both optical and acoustical plasmons move away from the boundary of the particle-hole continuum with a decrease in the electron density. We find that the introduction of XC corrections results in a significant enhancement of the transresistivity and qualitative changes in its temperature dependence. In particular, the large high-temperature plasmon peak that is present in the random phase approximation is found to disappear when the XC corrections are included. Our numerical results at low temperatures are in good agreement with the results of recent experiments by Kellogg et al. [Solid State Commun. 123, 515 (2002)].
\end{abstract}

DOI: 10.1103/PhysRevB.75.125321

PACS number(s): 73.40.-c, 71.45.Gm, 73.20.Mf, 73.63.Hs

\section{INTRODUCTION}

In a double-layer two-dimensional electron system (2DES) the additional degree of freedom associated with the layer index plays a key role in many recently discovered collective phenomena. ${ }^{1}$ Suffice it to mention the freshly provided evidence that in the quantum Hall regime, near the half filling of individual layers, strong electron-hole interlayer correlations cause Bose-Einstein condensation of excitons to occur in bilayer 2D electron and hole systems. ${ }^{2}$ In these structures, the ability to make separate electrical contacts to each layer has allowed the experimental detection of transport of neutral excitons as a counterflowing current of electrons and holes in two layers. The independent control of layers is also critical for the experimental realization of the frictional drag $^{3,4}$ which manifests itself under the condition that the second layer is an open circuit. A current driven along the first layer induces, via momentum transfer, a voltage in the second layer, which is measured experimentally. The drag effect complements and enriches the traditional experimental methods and in the last decade has proved to be a powerful tool to probe interlayer electron-electron $(e-e)$ interaction. $^{5}$

Recently the drag measurements have been extended to the limit of very low carrier concentrations. The dimensionless parameter $r_{s}=\sqrt{2} /\left(k_{F} a_{B}^{*}\right)$, which describes the carrier density $n$ and measures the strength of the electron-electron interaction, ${ }^{6}$ varies approximately from 10 to 20 in the experiment on hole samples by Pillarisetty et al. ${ }^{7,8}$ Here $k_{F}$ $=\sqrt{2 \pi n}$ is the Fermi wave vector, and $a_{B}^{*}=\hbar^{2} \kappa_{0} / m^{*} e^{2}$ is the effective Bohr radius, with $\kappa_{0}$ the static dielectric constant and $m^{*}$ the effective mass of the carriers; $a_{B}^{*}=9.79$ and $3.45 \mathrm{~nm}$, respectively, in the conduction $\left(m_{e}^{*}=0.067 m_{0}\right)$ and valence $\left(m_{h}^{*}=0.19 m_{0}\right)$ bands of GaAs. In the experiment on electron samples by Kellogg et al. ${ }^{9} r_{s}$ varies approximately from 2.5 to 4.5 and is appreciably smaller, owing to the small effective mass of the electrons. From a dimensional analysis it is clear, however, that in both types of samples, the Coulomb potential energy dominates the kinetic energy, and an adequate description of the drag cannot be provided by simple theories, which do not include the strong exchangecorrelation (XC) effects. Consistent with this expectation, both experimental groups ${ }^{7-9}$ have found that the measured drag rate far exceeds (by more than a factor 200 in the hole samples) the value obtained from simple Boltzmannequation calculations. ${ }^{10}$ In addition, systematic deviations are observed from the quadratic temperature dependence of the drag rate (the characteristic Fermi-liquid behavior of the Coulomb drag at low temperatures).

One might hope that the above features could be understood in the framework of the phonon-mediated drag mechanism. ${ }^{11-17}$ However, the phonon-mediated drag provides a dominant mechanism only in samples with large interlayer spacing where the absolute value of the measured drag rate is much smaller than that observed in these experiments. Moreover, the phonon mechanism is not supported by the measured density-ratio dependence of drag, which shows no peak at matched carrier densities in two layers, typical for that processes. Thus, both experimental groups ${ }^{7-9}$ conclude that in these dilute 2DESs the strong Coulomb interaction effects are mainly responsible for the observed new features.

The carrier interaction effects on drag have been addressed previously in several experimental ${ }^{18-20}$ and 
theoretical ${ }^{21-23}$ papers. While the theoretical prediction by Flensberg and $\mathrm{Hu}^{22}$ of a strong enhancement of drag by plasmons has been experimentally verified ${ }^{19}$ in high-density electron samples, important differences have been reported ${ }^{20}$ from the results obtained within the random phase approximation (RPA).

Lately, motivated by the recent set of low-density experiments, ${ }^{7-9}$ the drag resistivity has been calculated by Hwang et $a l .{ }^{24}$ and Yurtserver et al. ${ }^{25}$ Both works included only the exchange interaction effects in the static limit via $q$-dependent but $\omega$-independent local field factors (LFFs). In addition, in the adopted approximation the nondiagonal interlayer LFFs have been taken to be zero, ${ }^{24}$ while for the intralayer LFFs the simple Hubbard approximation has been used, which significantly underestimates the LFFs. As in the low-density regime the interparticle spacing in each layer becomes comparable with the interlayer separation, one should expect that interlayer correlations play an important role. At relatively high temperatures the dynamic XC effects are also critical. They can be especially important in the hole samples where, even at sufficiently low temperatures, the samples are effectively in the high-temperature regime because of the small Fermi energy.

In this paper we investigate the XC effects on the Coulomb drag in low-density $e$ - $e$ bilayers. The spectrum of plasmons of the coupled 2DESs and the temperature dependence of the drag resistivity are calculated in a broad range of temperatures. Our calculations include both intra- and interlayer $\mathrm{XC}$ effects. We propose a different approach, which employs dynamic XC kernels in the calculation of the bilayer plasmon spectra and of the plasmon-mediated drag, while the particlehole contribution to the drag is still calculated by means of static many-body LFFs. We find that the introduction of XC corrections results in a significant enhancement of the drag resistivity and qualitative changes in its temperature dependence. In particular, a large high-temperature plasmon peak that is present in the RPA disappears when the XC corrections are included. At low temperatures our numerical results are in good agreement with the experimental findings by Kellogg et al. ${ }^{9}$

The paper is organized as follows. In Sec. II we outline the theoretical formulation of the frictional drag and provide the main formulas for its calculation beyond the RPA. In Sec. III a method is developed to relate the spin-averaged dynamic XC kernels and static many-body LFFs of a bilayer 2DES to the spin-resolved XC kernels and LFFs of a single electron gas layer of the same total density. The results of our calculations are given in Secs. IV-VI. First we present the spectra of bilayer plasmons for different densities and discuss the effect of dynamic $\mathrm{XC}$ on the dispersion of each plasmon mode, comparing the dispersion curves with the corresponding curves obtained within the RPA. In Sec. V we study the temperature dependence of the drag resistivity at low densities within the RPA. Both plasmon and particlehole contributions to the drag are considered. In the next section we investigate the many-body XC effects on the drag resistivity for different densities and examine the distinctive features of the drag temperature dependence in comparison with the RPA. In the same Sec. VI we compare the obtained theoretical results with the experimental findings in low- density $e$-e bilayers. Finally, Sec. VII summarizes the main results of the paper.

\section{THEORETICAL FORMULATION}

We calculate the temperature dependence of the drag resistivity starting from the formula, obtained in the Kubo formalism, ${ }^{26}$

$$
\begin{aligned}
\rho_{D}= & \frac{\hbar^{2}}{2 e^{2} \sigma_{1} \sigma_{2} T A} \frac{d \sigma_{1}}{d n_{1}} \frac{d \sigma_{2}}{d n_{2}} \sum q_{\vec{q}} \int_{0}^{\infty} \frac{d \omega}{2 \pi}\left|W_{12}(q, \omega)\right|^{2} \\
& \times \frac{\operatorname{Im} \Pi_{1}^{0}(q, \omega) \operatorname{Im} \Pi_{2}^{0}(q, \omega)}{\sinh ^{2}(\hbar \omega / 2 T)},
\end{aligned}
$$

where $A$ is the normalization area, $\hbar \omega$ and $\hbar \vec{q}$ the transferred energy and in-plane momentum from layer 1 to layer 2, $W_{12}(q, \omega)$ the dynamically screened interlayer $e$-e interaction including the intra- and interlayer many-body XC effects of a bilayer $2 \mathrm{DES}, \Pi_{1,2}^{0}(q, \omega)$ the finite-temperature electron polarization function of an individual 2DES in the absence of interparticle Coulomb interaction. The imaginary and real parts of $\Pi_{1,2}^{0}(q, \omega)$ are obtained from the Maldague formula ${ }^{22,27}$ and are given by

$$
\begin{aligned}
\operatorname{Im} \Pi^{0}(q, \omega)= & g_{0} \frac{\sqrt{\pi t}}{4 x}\left[F_{-1 / 2}\left(\frac{\mu(t)-\zeta_{+}^{2}(x, y)}{t}\right)\right. \\
& \left.-F_{-1 / 2}\left(\frac{\mu(t)-\zeta_{-}^{2}(x, y)}{t}\right)\right]
\end{aligned}
$$

and

$$
\begin{aligned}
\operatorname{Re} \Pi^{0}(q, \omega)= & g_{0}\left(1-e^{-1 / t}-\frac{1}{2 x}\left\{M\left(t, \zeta_{+}^{2}(x, y)\right)\right.\right. \\
& \left.\left.-\operatorname{sgn}\left[\zeta_{-}(x, y)\right] M\left(t, \zeta_{-}^{2}(x, y)\right)\right\}\right),
\end{aligned}
$$

where we have introduced the following dimensionless quantities: $\quad t=T / \varepsilon_{F}, \quad x=q / 2 k_{F}, \quad y=\omega / 4 \varepsilon_{F}, \quad \mu(t)=t \ln \left(e^{1 / t}-1\right)$, $\zeta_{ \pm}(x, y)=y / x \pm x$ with $\varepsilon_{F}$ and $k_{F}$ being the Fermi energy and the Fermi wave vector. The function

$$
F_{-1 / 2}(u)=1 / \sqrt{\pi} \int_{0}^{\infty} d z / \sqrt{z}[\exp (z-u)+1]
$$

is the Fermi integral, the function

$$
M(t, u)=1 / 4 t \int_{0}^{u} d z \sqrt{u-z} / \cosh \{[z-\mu(t)] / 2 t\}^{2},
$$

and $g_{0}=m^{*} / \pi \hbar^{2}$ is the density of states of the noninteracting 2DES. Further we assume that in Eq. (1) the layer conductivities $\sigma_{1,2}$ depend linearly on the electron densities $n_{1,2}$. In general, this is an acceptable approximation for the conducting 2DES in the magnetic-field-free case. Deviations from the linear regime can occur at very low electron densities. Here we consider only the regime corresponding to $r_{s} \leqslant 5$; in this regime we expect the neglect of the corrections to the $\sigma_{1,2} \infty n_{1,2}$ to be a reasonable approximation. 
Our starting equation (1) is an approximation, strictly justifiable only to second order in the Coulomb interaction. The correct expression for the transresistivity involves a forceforce response function, which is a four-point response function and cannot be reduced, in general, to a product of twopoint response functions. In order to include the XC effects on the dynamically screened interlayer Coulomb interaction, in our calculations we have exploited an approximation scheme for $W_{12}$ proposed by Zheng and MacDonald ${ }^{28}$ (see also Ref. 23). Within this scheme an exact matrix Dyson equation for the Coulomb propagator, ${ }^{29} \hat{W}(q, \omega)$, is approximated by

$$
\hat{W}(q, \omega)=\hat{V}_{\text {eff }}(q, \omega)-\hat{V}_{\text {eff }}(q, \omega) \hat{\Pi}(q, \omega) \hat{V}_{\text {eff }}(q, \omega)
$$

with the full polarization function $\hat{\Pi}(q, \omega)$ defined in terms of the noninteracting polarization function $\hat{\Pi}_{0}(q, \omega)$ and unscreened effective Coulomb interactions $\hat{V}_{\text {eff }}(q, \omega)$ as

$$
\hat{\Pi}(q, \omega)=\frac{\hat{\Pi}_{0}(q, \omega)}{1+\hat{V}_{\mathrm{eff}}(q, \omega) \hat{\Pi}_{0}(q, \omega)} .
$$

Here all quantities are $2 \times 2$ matrices. The effective Coulomb interactions $\hat{V}_{\text {eff }}$ (whose explicit form is given below) build in Eqs. (6) and (7) the vertex corrections as sums of ladder diagrams evaluated within a "local approximation." 30 Thus, the nondiagonal interaction matrix element $W_{12}(q, \omega)$ is given by

$$
W_{12}(q, \omega)=\frac{V_{\mathrm{eff}, 12}(q, \omega)}{\varepsilon_{\mathrm{bi}}(q, \omega)}
$$

with the bilayer screening function

$$
\varepsilon_{\mathrm{bi}}(q, \omega)=\varepsilon_{1}(q, \omega) \varepsilon_{2}(q, \omega)-V_{\mathrm{eff}, 12}(q, \omega)^{2} \Pi_{1}^{0}(q, \omega) \Pi_{2}^{0}(q, \omega) .
$$

Here we introduce the screening functions of individual layers

$$
\varepsilon_{1,2}(q, \omega)=1+V_{\mathrm{eff}, 11,22}(q, \omega) \Pi_{1,2}^{0}(q, \omega) .
$$

The approximation given by Eqs. (6)-(10) accounts for the effect of dynamical screening of the Coulomb potential via the bilayer screening function $\varepsilon_{\mathrm{bi}}(q, \omega)$, and includes the correlations between the test electron and the induced charge via the effective Coulomb interactions $\hat{V}_{\text {eff }}(q, \omega)$. In this approximation XC LFFs manifest themselves only via the intra- and interlayer unscreened effective interactions

$$
V_{\mathrm{eff}, i j}(q, \omega)=v(q)\left[1-G_{\mathrm{XC}, i j}(q, \omega)\right] F_{i j}(q d, q \Lambda)
$$

where the intra- and interlayer LFFs $G_{\mathrm{XC}, i j}(q, \omega)$ decrease effectively the bare Coulomb interaction $v(q)=2 \pi e^{2} / \kappa_{0} q$ by a factor of $1-G_{\mathrm{XC}, i j}(q, \omega)(i, j=1,2$ are the layer indices). Notice that the RPA is recovered if all the LFFs in Eq. (8) are set to zero.

Further we assume that the electrons in each layer are confined in a square well potential of infinite height and that scattering processes take place only within the lowest subband of each well. Assuming $\rho_{1}(z)=(2 / d) \sin (\pi z / d)^{2}$ and
$\rho_{2}(z)=\rho_{1}(z+\Lambda)$ as explicit forms for the electron density profiles in layers 1 and 2 , respectively, one obtains ${ }^{10}$ for the corresponding intra- and interlayer form factors [see Eq. (11)]

$$
F_{11}(\eta, \xi)=\frac{8 \pi^{2}+3 \eta^{2}}{\eta\left(4 \pi^{2}+\eta^{2}\right)}-\frac{32 \pi^{4}\left(1-e^{-\eta}\right)}{\eta^{2}\left(4 \pi^{2}+\eta^{2}\right)^{2}}
$$

and

$$
F_{12}(\eta, \xi)=\frac{64 \pi^{4} \sinh (\eta / 2)^{2}}{\eta^{2}\left(4 \pi^{2}+\eta^{2}\right)^{2}} e^{-\xi},
$$

where $\Lambda$ is the center-to-center interlayer separation and $d$ the width of quantum wells.

\section{DYNAMIC XC KERNEL AND STATIC LFFS}

In general the local field factors of a bilayer system, $\widetilde{G}_{i j}^{\mu \nu}(q, \omega)$, depend on four indices: the spin indices $\mu$ and $\nu$, taking values $\uparrow$ and $\downarrow$, and the layer indices $i$ and $j$, taking values 1 and 2. But the LFFs that appear in Eq. (11), and hence in the effective interaction $W_{12}$, are averaged over the spin indices, at least as long as we assume that our bilayer is not spin polarized. Thus we have

$$
G_{\mathrm{XC}, i j}(q, \omega) \equiv \frac{1}{4} \sum_{\mu \nu} \widetilde{G}_{i j}^{\mu \nu}(q, \omega) .
$$

Unfortunately, reliable calculations of the LFFs for bilayers (for example, by the diffusion Monte Carlo method) have not been done to date. In order to proceed in the absence of this crucial information we propose an alternative approach, in which the spin-averaged LFFs of the bilayer are expressed in terms of the spin-resolved LFFs of a single electron layer of the same total density $n=n_{1}+n_{2}$ - the reference monolayer. We suggest exploiting the analogy between the spin index of the reference monolayer and the pseudospin (layer) index of the original bilayer, neglecting the spin index in the bilayer altogether. We thus map the layer index (in the bilayer) onto the spin index in the monolayer and represent the intra- and interlayer LFFs of the bilayer in terms of the spinchannel and charge-channel LFFs of the reference monolayer, denoted, respectively, by $G_{\mathrm{m}}^{-}(q, \omega)$ and $G_{\mathrm{m}}^{+}(q, \omega)$ in the following manner:

$$
\begin{aligned}
G_{\mathrm{XC}, 11}(q, \omega) & =G_{\mathrm{m}}^{+}(q, \omega)+G_{\mathrm{m}}^{-}(q, \omega), \\
G_{\mathrm{XC}, 12}(q, \omega) & =G_{\mathrm{m}}^{+}(q, \omega)-G_{\mathrm{m}}^{-}(q, \omega) .
\end{aligned}
$$

Of course, this simple representation is possible only in the symmetric case, $n_{1}=n_{2}$, when we have $G_{\mathrm{XC}, 11}(q, \omega)$ $=G_{\mathrm{XC}, 22}(q, \omega)$ and $G_{\mathrm{XC}, 12}(q, \omega)=G_{\mathrm{XC}, 21}(q, \omega)$.

It is evident that Eq. (15) is not exact: for one thing, the interaction between electrons in different layers is different from the interaction between two electron in the same layer-a difference that does not exist between parallel- and antiparallel-spin electrons in the reference monolayer. Subtle differences persist even in the limit of zero interlayer separation. In the reference monolayer the Pauli exclusion prin- 


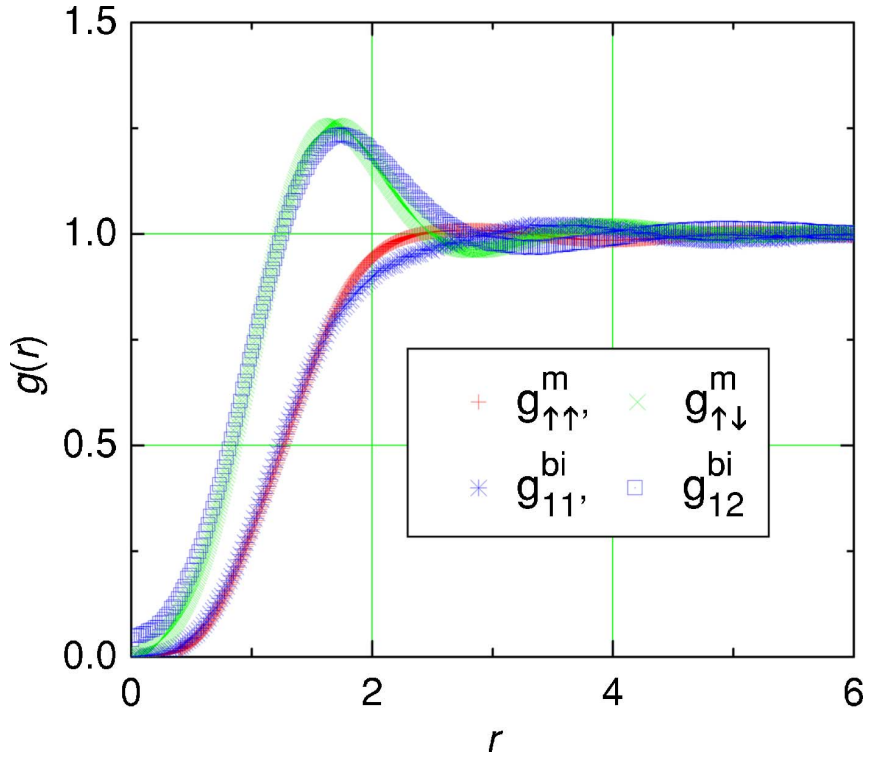

FIG. 1. (Color online) Bilayer intra- and interlayer paircorrelation functions $g_{11}^{\mathrm{bi}}(r)$ and $g_{12}^{\mathrm{bi}}(r)$ and spin-resolved paircorrelation functions $g_{\uparrow \uparrow}^{\mathrm{m}}(r)$ and $g_{\uparrow \downarrow}^{\mathrm{m}}(r)$ for a monolayer as directly obtained from the quantum Monte Carlo simulations, respectively, from Refs. 31 and 32. The total $r_{s}=7.07$ in both the monolayer and bilayer. It corresponds to the in-layer $r_{s}^{\text {in layer }}=10$ of the bilayer. The interlayer spacing of the bilayer is $\Lambda=0.2 r_{s}^{\text {in layer }} a_{B}^{*}$.

ciple keeps two electrons of the same spin orientation from coming close to each other; but in the bilayer two electrons in the same layer (i.e., with parallel isospin) can come together provided their real spins are opposite.

In spite of these difficulties we find that the approximation (15) is quite reasonable at small interlayer separations. We get a good idea of the electronic correlations in a system by examining its pair-distribution functions. In Fig. 1 we compare the spin-averaged pair-distribution functions of a bilayer, $g_{11}^{\text {bi }}(r)$ (intralayer) and $g_{12}^{\text {bi }}(r)$ (interlayer), as obtained from the diffusion Monte Carlo simulations, ${ }^{31}$ with the spinresolved pair-distribution functions $g_{\uparrow \uparrow}^{\mathrm{m}}(r)$ and $g_{\uparrow \downarrow}^{\mathrm{m}}(r)$ of the reference monolayer. ${ }^{32}$ It is seen that there is an overall good agreement between the corresponding distribution functions, i.e., $g_{11}^{\text {bi }}$ agrees with $g_{\uparrow \uparrow}^{\mathrm{m}}$ and $g_{12}^{\mathrm{bi}}$ with $g_{\uparrow \downarrow}^{\mathrm{m}}$. This agreement provides much support for the use of Eq. (15). As $\Lambda$ increases, the agreement between the bilayer pair-distribution function $g_{12}^{\text {bi }}(r)$ and the monolayer pair-distribution function $g_{\uparrow \downarrow}^{\mathrm{m}}(r)$ will deteriorate. However, the disagreement between the interlayer correlations and the unlike-spin correlation in the reference monolayer is more severe at small $r$ than at large $r{ }^{31}$ The pair-distribution function at small values of $r$, in turn, should control the behavior of the LFFs at large wave vector $q \gg 2 k_{F}$, while the main contribution to the Coulomb drag, at low densities, comes from scattering processes with $q$ near $2 k_{F}$. Thus, we see that our approach could be justified for the problem at hand, even when the interlayer spacing is less than or comparable to the in-layer interparticle distance, $\Lambda \lesssim r_{s} a_{B}^{*}$, which is the case in the experiments of interest here.

Let us now come to the discussion of the LFFs in the reference monolayer. Here we have to introduce further ap- proximations. When treating the plasmon contribution to the Coulomb drag in our calculations we approximate the dynamic XC kernels $f_{\mathrm{XC}, i j}(q, \omega) \equiv-v(q) G_{\mathrm{XC}, i j}(q, \omega)$ by their long-wavelength limits and employ the dynamic spin-spin and charge-charge XC kernels, evaluated by Qian and Vignale in Refs. 34 and 35. In this way we include the frequency dependence of the dynamic charge-charge LFFs, while their wave vector dependence is implicitly assumed to be linear so that the charge-charge XC kernel is independent of $q$. This is a reasonable approximation in view of the nearly linear behavior of the exact static LFFs versus $q$ in the single layer. ${ }^{6}$ On the other hand, the spin-spin dynamic XC kernel is strongly dependent on $q$ and diverges as $q^{-2}$ at small wave vectors. ${ }^{36}$ Thus, the intra- and interlayer dynamic XC kernels in the long-wavelength limit are represented as

$$
f_{\mathrm{XC}, i j}(q, \omega)=B^{+}(\omega)+\left(2 \delta_{i j}-1\right)\left(\frac{A(\omega)}{q^{2}}+B^{-}(\omega)\right)
$$

where $A(\omega)$ and $B^{ \pm}(\omega)$ are finite functions of $\omega$ and have been calculated to the leading order in the Coulomb interaction in Refs. 34 and 35. The authors of this paper have evaluated the four-vertex diagrams in the expansion of the response function of individual 2DESs in the low-frequency and high-density limit. The interpolation formulas for $\operatorname{Im} A(\omega)$ and $\operatorname{Im} B^{ \pm}(\omega)$ have been constructed by combining the results for the imaginary parts with sum rules and previously known exact behaviors in the high-frequency limit. ${ }^{37}$ Thus, having the imaginary parts of $A(\omega)$ and $B^{ \pm}(\omega)$, we obtain the corresponding real parts from the KramersKroning relations.

Figures 2 and 3 show plots of the imaginary and real parts of the intra- and interlayer $\mathrm{XC}$ kernels $f_{\mathrm{XC}, 11}(q, \omega)$ and $f_{\mathrm{XC}, 12}(q, \omega)$, which we actually use in our calculations of the spectrum of bilayer plasmons and of the Coulomb drag mediated by these plasmons. Notice that the kernels are calculated for the shown values of $r_{s}=2,3$, and 5, which are obtained for the total number of electrons $n$ in a bilayer. The corresponding in-layer values of $r_{s}^{\text {in layer }}$, calculated for $n / 2$ electrons in individual layers, are $r_{s}^{\text {in layer }}=2 \sqrt{2}, 3 \sqrt{2}$, and $5 \sqrt{2}$. At finite frequencies, the term in Eq. (16), related to $A(\omega)$, dominates in both the short- and long-wavelength limits. The terms related to $B^{ \pm}(\omega)$ make essential contributions to $f_{\mathrm{XC}, i j}(q, \omega)$ only in the small- $\omega$ region, where $A(\omega)$ vanishes as $\omega^{2}$.

The frequency dependence of the LFFs in the particlehole continuum (PHC) region (finite wave vector, low frequency) is still largely unknown. For this reason, in evaluating the particle-hole contribution to the drag resistivity, we use the static limit of the LFFs. We take advantage of the analytical expressions recently obtained for $G_{\mathrm{XC}}^{-}(q)$ and $G_{\mathrm{XC}}^{+}(q)$, LFFs, ${ }^{38}$ which accurately reproduce the diffusion Monte Carlo data, ${ }^{33}$ as well as the exact asymptotic behaviors in the large- and small- $q$ limits. Notice that both the dynamic XC kernels ${ }^{34,35}$ and the static LFFs, ${ }^{38}$ which we have used in our calculations, are evaluated at $T=0$ for an ideal 2DES of zero width. At present there are no calculations of the LFFs of a two-dimensional system that take into account the finite width of the well and/or the temperature 

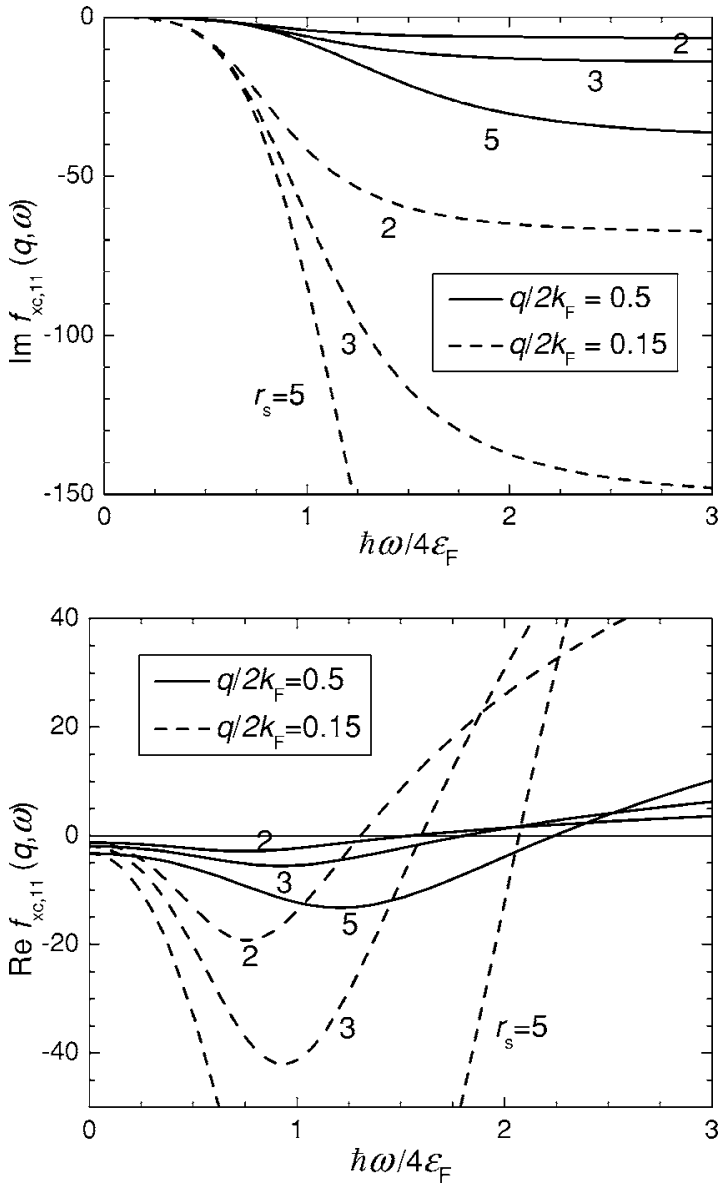

FIG. 2. Imaginary part (figure on top) and real part (figure below) of the intralayer dynamic XC kernel $f_{\mathrm{XC}, 11}(q, \omega)$ of a bilayer 2DES as a function of $\omega$. The kernels are shown for the total $r_{s}$ $=2,3$, and 5 and for two different values of the wave vector $q$ in units of $g_{0}^{-1}$ where $g_{0}=m^{*} / \pi \hbar^{2}$ is the density of states for the noninteracting $2 \mathrm{DES}$.

dependence. We expect, however, that the temperature dependence of the LFFs should play a relatively minor role in comparison to the temperature dependence that we explicitly include in the Fermi factors and the noninteracting polarization functions. Similarly, the effect of the finite width of the well should largely be taken care of by our use of the form factors $F_{i j}$ in Eq. (11). In Fig. 4 we plot the intra- and interlayer LFFs $G_{\mathrm{XC}, i j}(q)$ which we actually use in our calculations of the drag resistivity in the PHC region. For all the values of $r_{s}$ shown in the figure, the intralayer LFFs $G_{\mathrm{XC}, 11}(q)$ become significantly larger than the LFF in the Hubbard approximation, $G_{\mathrm{H}}(x)=x / \sqrt{1+4 x^{2}}$ when $x$ is close to unity. This makes the effective intralayer interaction weak in the region near $x=1$ and results in a weak in-plane screening effect. This effect becomes especially important in the regime of low-density bilayers where the main contribution to drag comes from the large-angle scattering processes with $x \approx 1$. In Fig. 4 we also see that although $G_{\mathrm{XC}, 12}(q)$ is smaller than $G_{\mathrm{XC}, 11}(q)$, the two LFFs are of the same order of magnitude.
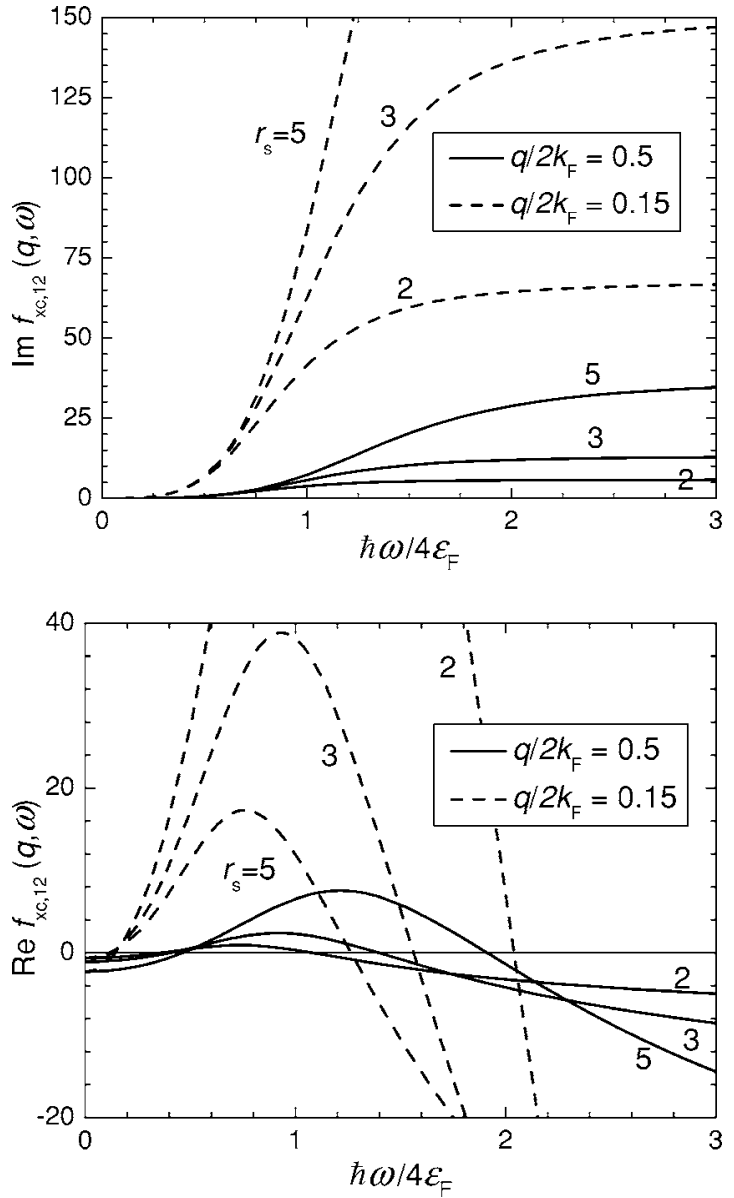

FIG. 3. Imaginary part (figure on top) and real part (figure below) of the interlayer dynamic XC kernel $f_{\mathrm{XC}, 12}(q, \omega)$ of a bilayer 2DES as a function of $\omega$. The kernels are shown for the total $r_{s}$ $=2,3$, and 5 and for two different values of the wave vector $q$ in units of $g_{0}^{-1}$ where $g_{0}=m^{*} / \pi \hbar^{2}$ is the density of states of the noninteracting 2DES.

\section{BILAYER PLASMON DISPERSIONS: EFFECT OF DYNAMIC XC}

Below we consider the symmetric bilayer 2DES $\left(n_{1}=n_{2}\right)$ with interlayer spacing $\Lambda=28 \mathrm{~nm}$ and quantum well width $d=18 \mathrm{~nm}$, which correspond to the experimental situation of Ref. 9.

In this section we calculate the spectrum of bilayer plasmons for the total $r_{s}=2$ and $3\left(r_{s}^{\text {in layer }}=2 \sqrt{2}\right.$ and $\left.3 \sqrt{2}\right)$ both within the RPA and beyond it, taking into account the dynamic XC effects through the intra- and interlayer XC kernels. In symmetric systems the bilayer screening function is represented as

$$
\varepsilon_{\mathrm{bi}}(q, \omega)=\varepsilon_{+}(q, \omega) \varepsilon_{-}(q, \omega),
$$

where

$$
\varepsilon_{ \pm}(q, \omega)=1+V_{ \pm}(q, \omega) \Pi^{0}(q, \omega),
$$

with $V_{ \pm}(q, \omega)=V_{\text {eff, } 11}(q, \omega) \pm V_{\text {eff,12 }}(q, \omega)$. The spectrum of the collective excitations is obtained from the zeros of the 

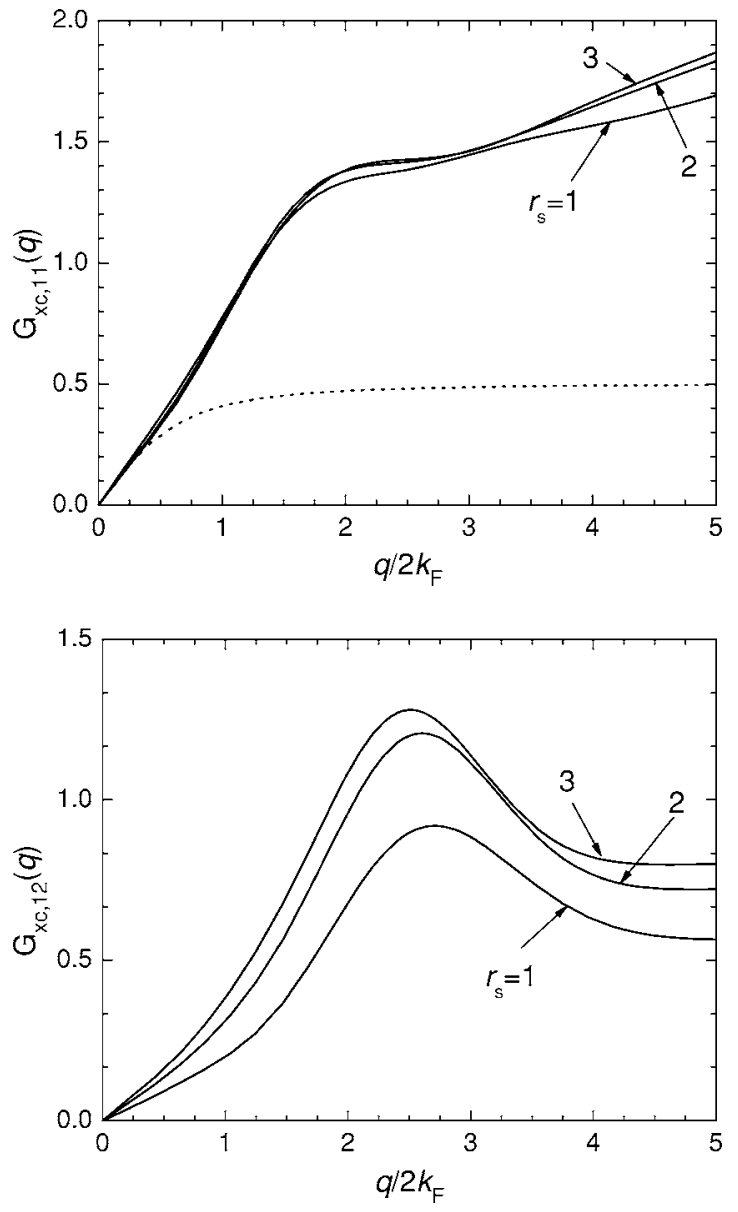

FIG. 4. Static many-body LFFs of a double-layer 2DES. The intralayer $G_{\mathrm{XC}, 11}$ (figure on top) and the interlayer $G_{\mathrm{XC}, 12}$ (figure below) are plotted as a function of wave vector $q$ for equal electron densities in the two layers corresponding to the total $r_{s}=1,2$, and 3 . The dotted curve shows the LFF $G_{\mathrm{H}}(x)$ in the Hubbard approximation.

real part of the bilayer screening function so the dispersion is obtained from the solution of the equation

$$
\operatorname{Re} \varepsilon_{ \pm}\left(q, \omega_{\text {op }, \text { ac }}(q)\right)=0 .
$$

The imaginary part of the screening function describes the damping of the collective modes. In this approach the imaginary parts of the dynamic XC kernels are finite at finite frequencies and we obtain that even at zero temperature the collective excitations are Landau damped outside the $T=0$ $\mathrm{PHC}$ region. Also, we use the finite-temperature forms for the polarization function $\Pi^{0}(q, \omega)$ which itself has a twofold effect on the plasmon spectrum. The finite-temperature contribution to the imaginary part of $\Pi^{0}(q, \omega)$ outside the $T=0$ $\mathrm{PHC}$ region enhances the damping of the collective modes, while temperature-induced changes in the real part of $\Pi^{0}(q, \omega)$ modify the domain of existence of the collective excitations.

In Fig. 5 we plot the plasmon spectra at zero and finite temperatures for the total $r_{s}=3$, based on the above Eq. (19). The spectra are calculated within the RPA and beyond it taking into account the full intra- and interlayer XC kernels.
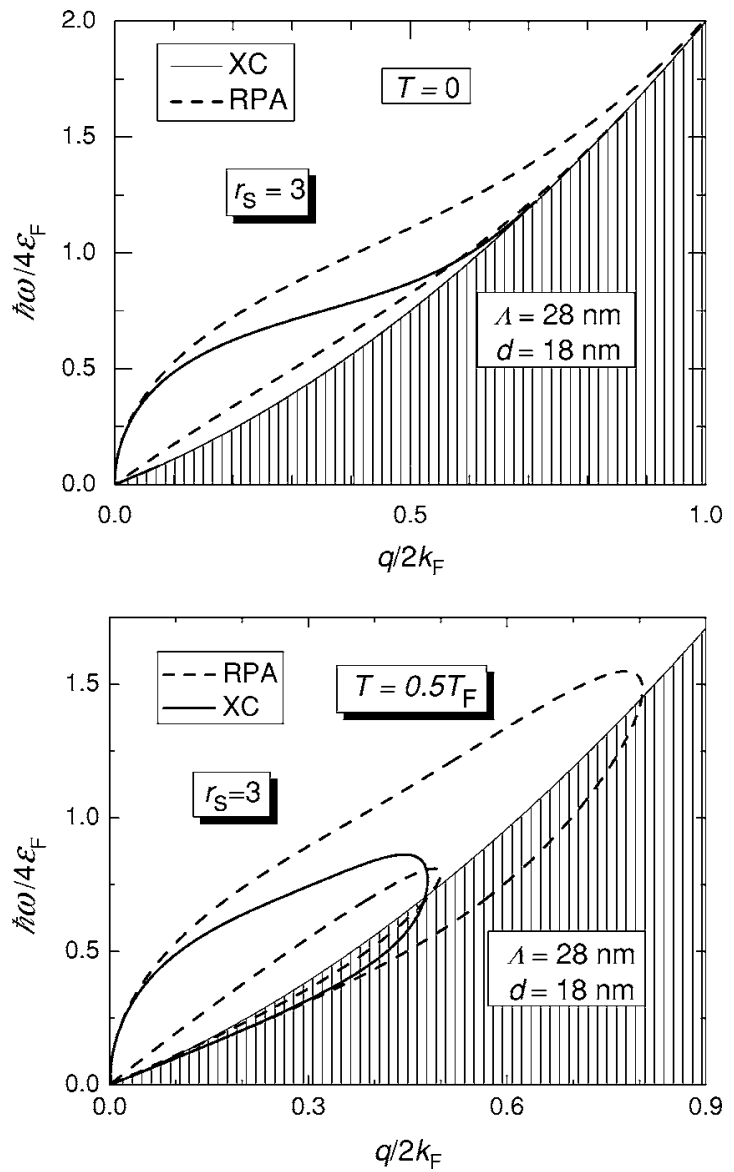

FIG. 5. Bilayer plasmon spectra at zero temperature (figure on top) and at finite temperature $T=0.5 T_{F}$ (figure below) for the total $r_{s}=3$. The hatched area shows the PHC region at $T=0$. The dispersion curves are calculated within the RPA (dashed curves) and beyond the RPA, taking into account the full intra- and interlayer dynamic XC corrections (solid curves).

The upper-lying "optical" branches in both approximations exhibit the expected square-root $\sim \sqrt{q}$ dispersion in the longwavelength limit, while the lower-lying "acoustical" branches have linear $\sim q$ dispersion. The inclusion of the dynamic XC kernels forces both the optical and acoustical branches to enter the PHC at substantially smaller values of $q$. At finite $T$ the dispersions of collective excitations show the same behavior in the long-wavelength limit while, at the upper edge of the wave vector interval in which the collective excitations exist, the group velocity of each type of plasmon, independently of the approximation used, becomes infinite.

Figure 6 shows that, as the electron density decreases, both the optical and the acoustical plasmon dispersions, calculated within the RPA, move away from the boundary of the PHC. We find, however, that the many-body effects beyond the RPA result in a new tendency, namely, the optical and the acoustical modes repel each other when $r_{s}$ increases so that the acoustical branch becomes closer to the boundary of the PHC while the optical branch is still pushed away from it. As we see below, all these changes, induced by the dynamic many-body effects, are directly reflected in the temperature dependence of the plasmon-mediated Coulomb drag. 

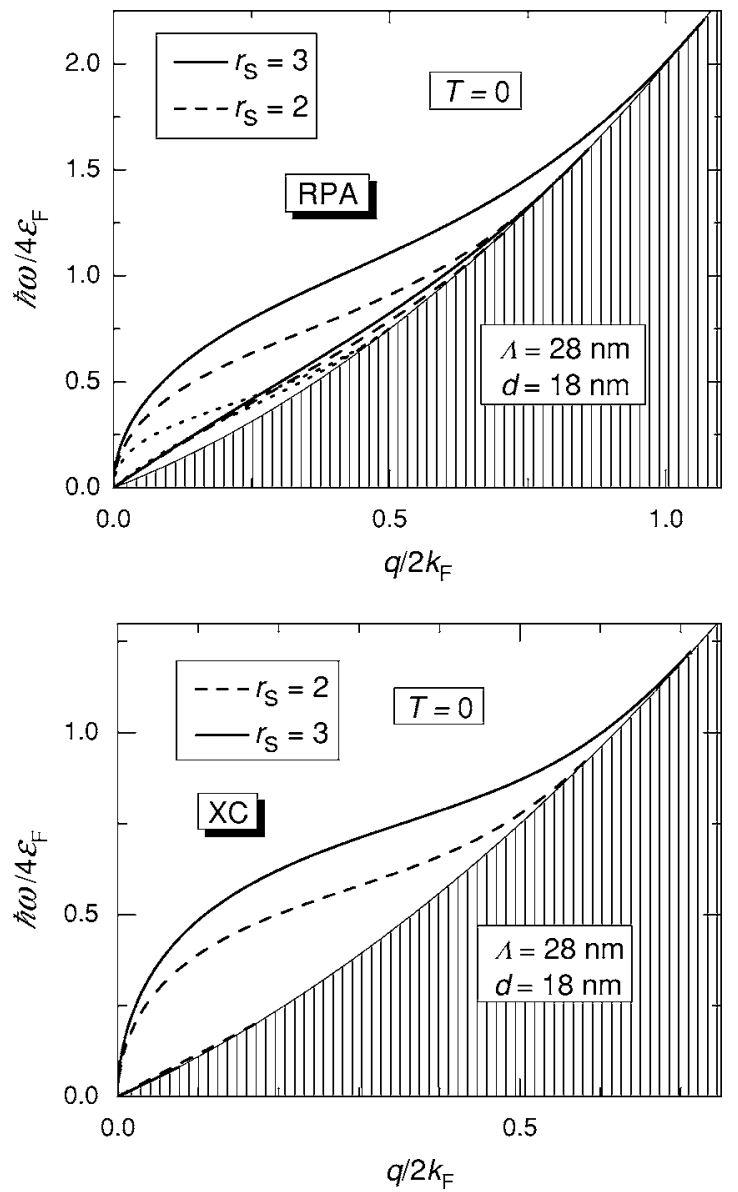

FIG. 6. Bilayer plasmon spectra at zero temperature. The hatched area shows the PHC region at $T=0$. The dispersion curves are calculated within the RPA (figure on top) and beyond the RPA by taking into account the effects of intra- and interlayer dynamic $\mathrm{XC}$ (figure below). The solid and dashed curves correspond to the values of the total $r_{s}=2$ and 3 , respectively. The dotted curves reproduce the plasmon dispersions from Ref. 22, calculated within the RPA for high-density, $n=1.5 \times 10^{11} \mathrm{~cm}^{-2}$, bilayer samples with the interlayer separation $k_{F} \Lambda=4$.

\section{DRAG RESISTIVITY WITHIN THE RPA}

First we present RPA-based calculations of the drag due to the exchange of plasmons in low-density bilayers. In Fig. 7 the transresistivity [scaled by the factor $\left(T / T_{F}\right)^{2}$ ] is plotted as a function of temperature for five different in-layer densities corresponding to $r_{s}^{\text {in layer }}=2,2 \sqrt{2}, 3,3 \sqrt{2}$, and 5 . Although the magnitude of the drag resistivity increases with $r_{s}$, it is seen that within the RPA the qualitative behavior of the plasmon-mediated drag does not undergo strong changes when the electron density decreases, and it resembles the behavior at high densities. ${ }^{22}$ The behavior of the transresistivity versus temperature exhibits an upturn at low temperatures near $0.2 T_{F}$ and a plasmon enhancement peak at higher temperatures. At low temperature the transresistivity decreases slightly with decreasing density. In contrast to this, at high temperatures the height of the transresistivity peak increases approximately linearly with $r_{s}^{\text {in layer }}$ in the range 2 $<r_{s}^{\text {in layer }}<5$. As seen in Fig. 7 both the upturn temperature

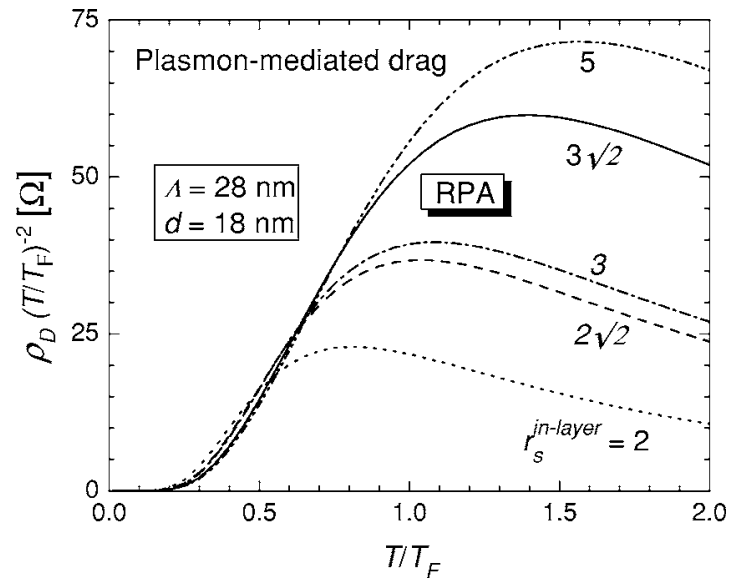

FIG. 7. Plasmon-mediated drag resistivity as a function of scaled temperature within the RPA. The curves from bottom to top correspond to the in-layer $r_{s}^{\text {in layer }}=\sqrt{2}, 2,2 \sqrt{2}, 3,3 \sqrt{2}$, and 5 .

of the plasmon-mediated drag and the position of the plasmon enhancement peak increase with $r_{s}^{\text {in layer }}$. This is consistent with the above discussion of the plasmon spectra, since in the RPA the plasmon dispersion curves for both modes move away from the PHC when the density decreases. Notice, however, that the absolute value of the upturn temperature and the position of the plasmon-enhancement peak (not scaled by $T_{F}$ ) show the opposite tendency and decrease with increasing $r_{s}^{\text {in layer }}$. This is due to the fact that the Fermi temperature $T_{F}$ decreases quadratically with increasing $r_{s}^{\text {in layer }}$.

In Fig. 8 we plot the total transresistivity, due to exchange of both plasmons and particle-hole excitations, against the part of the transresistivity that is due only to exchange of

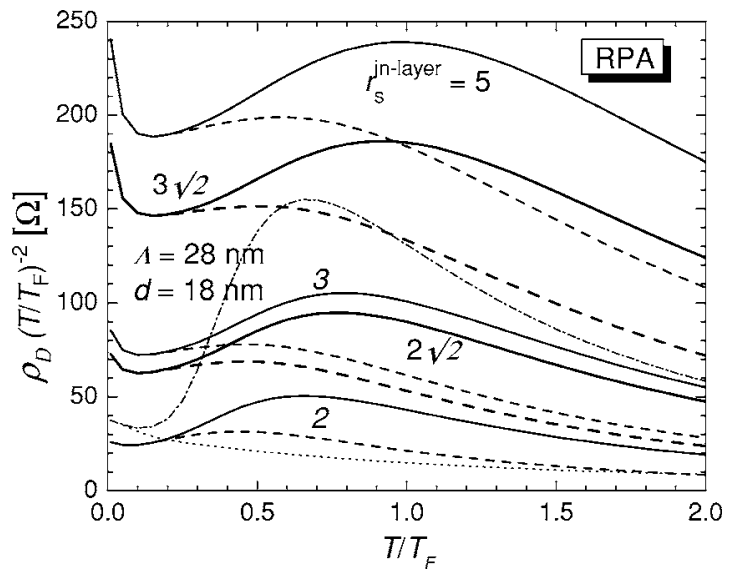

FIG. 8. Coulomb drag resistivity as a function of scaled temperature within the RPA for the in-layer $r_{s}=2,2 \sqrt{2}, 3,3 \sqrt{2}$, and 5 shown, respectively, from bottom to top. The solid curves correspond to the total drag, mediated both by plasmon and by particlehole excitations, while the dashed curves represent only the particle-hole contribution. The dash-dotted and dotted curves are for the situation corresponding to the first drag measurements of Ref. 39: they are rescaled by a factor of 20 , and represent, respectively, the total drag and the drag due to exchange of particle-hole excitations only. The latter is calculated within the static screening approximation. 
particle-hole excitations within the RPA. One can see that for $r_{s}=2,2 \sqrt{2}, 3,3 \sqrt{2}$, and 5 , when the density decreases, the contribution to the drag made by particle-hole excitations increases faster than the plasmon contribution to drag. However, the plasmon contribution remains essential in all cases and results in the plasmon-enhancement peaks, clearly seen in the behavior of the scaled transresistivity versus temperature.

The dotted and dash-dotted curves in Fig. 8 reproduce, respectively, the calculations by Jauho and Smith of the particle-hole contribution to the transresistivity based on the static screening approximation ${ }^{10}$ and by Flensberg and $\mathrm{Hu}$ of the plasmon-enhanced $\mathrm{drag}^{22}$ in the dynamic RPA. These curves correspond to the situation of the first drag experiment $^{39}$ on high-density samples with $n=1.5$ $\times 10^{11} \mathrm{~cm}^{-2}$ and with a large center-to-center inter-layer spacing $\Lambda=37.5 \mathrm{~nm}$. Notice that the plasmon-enhancement peak is most pronounced in Fig. 8. However, this strong effect is mainly caused by the large interlayer separation which exceeds significantly the effective spacing of the bilayers in the experiment in Ref. 9 for which the solid lines are calculated. As long as the plasmon-mediated drag depends more weakly on interlayer spacing than the particlehole contribution to drag, ${ }^{22}$ the plasmon-enhancement peak is more pronounced for the dotted curves.

\section{DRAG RESISTIVITY BEYOND THE RPA}

\section{A. The effect of dynamic XC on plasmon-mediated drag}

In this section we include the intra- and interlayer dynamic XC within the scheme (6)-(11) and study their effect on the plasmon-mediated drag by comparing the obtained results with those of RPA-based calculations. In Fig. 9 the scaled transresistivity as a function of temperature is shown for three equal-density bilayer systems with the total $r_{s}=1,2$, and 3 corresponding to the in-layer $r_{s}^{\text {in layer }}=\sqrt{2}, 2 \sqrt{2}$, and $3 \sqrt{2}$. It is seen that the upturn temperature in the scaled transresistivity essentially decreases when the XC effects are included. Despite slight differences, in both cases of $r_{s}^{\text {in layer }}$ $=\sqrt{2}$ and $2 \sqrt{2}$ the plasmons begin to contribute heavily to the interlayer $e-e$ interaction at temperatures less than $0.1 T_{F}$. At $r_{s}^{\text {in layer }}=3 \sqrt{2}$ the effect becomes even stronger. In contrast, according to the RPA calculation, the upturn of the plasmonmediated drag should be about $0.2 T_{F}$. This difference is explained by the changes in the spectrum of bilayer plasmons induced by the dynamic XC effects and discussed in the previous section. At low temperature the plasmon-mediated drag is mainly determined by the acoustical plasmons, whose frequency is reduced by the dynamic XC: this makes the acoustic plasmons easier to excite thermally, and causes their contribution to the Coulomb drag to be larger than in RPA.

When temperature increases, the average energy of plasmons that mediate the drag increases. Hence, the plasmon damping, caused by dynamic $\mathrm{XC}$ effects, becomes stronger with a consequent reduction in the drag transresistivity. Thus, at temperatures around $0.3 T_{F}$ the plasmon-mediated drag obtained within the RPA exceeds the drag which takes into account the XC effects.
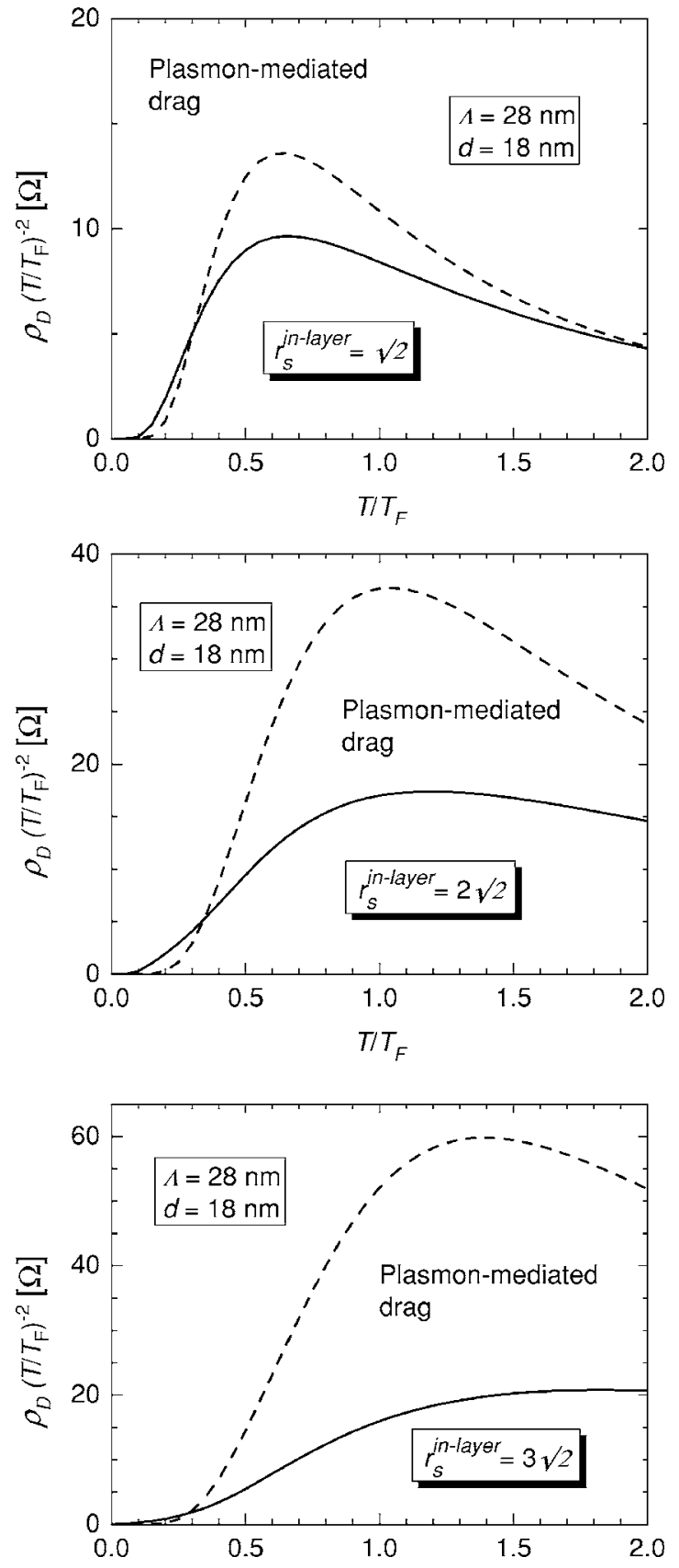

FIG. 9. Dynamic XC corrections to the plasmon-mediated drag for the in-layer $r_{s}^{\text {in layer }}=\sqrt{2}, 2 \sqrt{2}$, and $3 \sqrt{2}$. The scaled transresistivity vs temperature is shown within the RPA (dashed curves) and beyond the RPA taking into account the intra- and interlayer XC effects (solid curves).

At still higher temperatures the plasmon-mediated drag shows a broad peak both within the RPA and beyond it. The position of this peak is determined mainly by the position of the optical plasmons insofar as at such high temperatures the acoustical plasmons are heavily damped and merged into the PHC. As seen from Fig. 9 the peak position is at higher temperature at the lower density, and it changes slightly depending on the chosen approximation. These observations are again consistent with the behavior of the plasmon spectra in Figs. 5 and 6. The main contribution to the plasmon- 


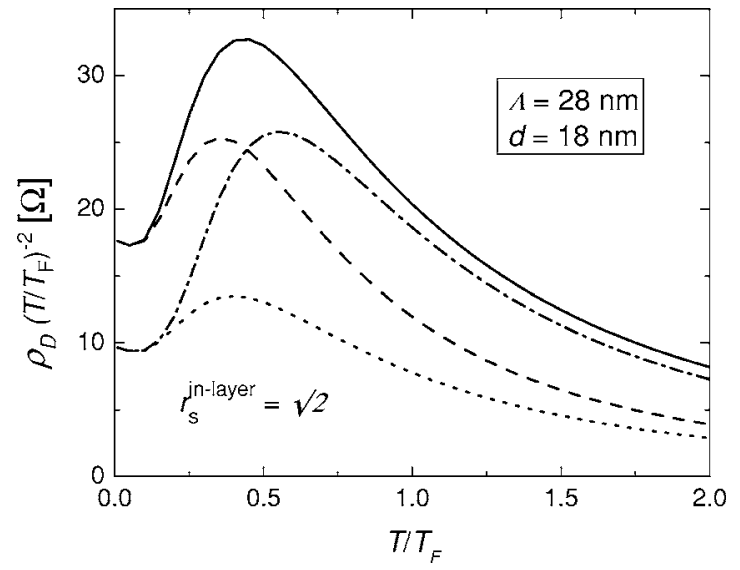

FIG. 10. The drag resistivity vs temperature for the in-layer $r_{s}^{\text {in layer }}=\sqrt{2}$. The solid and dashed curves represent the total transresistivity and the separate particle-hole contribution to the drag resistivity with the full intra- and interlayer XC corrections. The dashdotted and dotted curves are, respectively, the total transresistivity and the particle-hole contribution to the drag calculated within the RPA.

mediated drag is made by the plasmons with energies $\hbar \omega$ $\sim T$ while the scaled transresistivity peaks occur at temperatures $T \leq T_{F}$. Therefore, as seen in Figs. 5 and 6, at such small values of $\hbar \omega / 4 \varepsilon_{F} \leq 0.25$, the positions of the optical plasmons, and hence the positions of the peak in the plasmon contribution to drag in Fig. 9, remain approximately unaffected. Notice also that at even higher temperatures the differences in the magnitude of plasmon-mediated drag in the different approximations diminishes with increasing $T$.

\section{B. The combined effect of dynamic and static XC on the total drag}

In this section we investigate the combined effect of the dynamic and static many-body XC on the total drag, mediated both by plasmon and by particle-hole excitations. In Fig. 10 the drag resistivity is plotted vs temperature within and beyond the RPA for the total $r_{s}=1\left(r_{s}^{\text {in layer }}=\sqrt{2}\right)$. It is seen that in both approximations the particle-hole contribution to the scaled drag resistivity first shows a slight dip followed by a peak at higher temperatures. In the RPA the plasmon contribution to drag enhances this peak and shifts it to even higher temperatures so that the total transresistivity shows a pronounced peak approximately at the position where the plasmon contribution has a peak. As seen from Fig. 10 the introduction of the static exchange-controlled LFFs increases the peak height of the particle-hole contribution to the drag and shifts the peak toward lower temperatures. On the other hand, the plasmon-mediated drag is moderately suppressed by the dynamic $\mathrm{XC}$ corrections as discussed in the previous subsection (cf. Fig. 9). Thus, the resulting peak in the graph of the total transresistivity vs temperature becomes smaller at relatively low temperatures, while at high temperatures the drag rate shows a monotonic decrease in $T$.

The described quantitative and qualitative differences in the behavior of the scaled total transresistivity within and
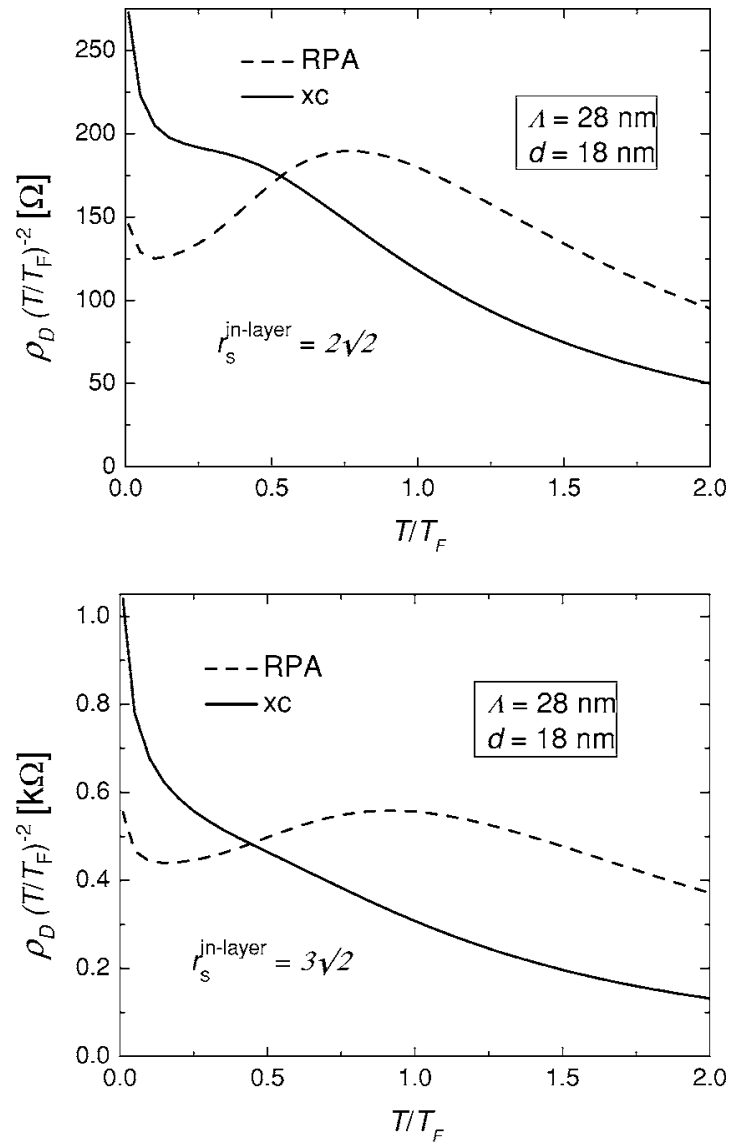

FIG. 11. The full XC corrections to the total transresistivity for the in-layer $r_{s}^{\text {in layer }}=2 \sqrt{2}$ and $3 \sqrt{2}$. The dashed and solid curves represent the scaled transresistivity vs temperature, calculated, respectively, within and beyond the RPA. The RPA data are multiplied by factors of 3 and 6 , respectively, for $r_{s}^{\text {in layer }}=2 \sqrt{2}$ and $3 \sqrt{2}$.

beyond the RPA becomes more pronounced at lower densities. As seen from Fig. 11 for the in-layer $r_{s}^{\text {in layer }}=2 \sqrt{2}$ and $3 \sqrt{2}$ the total transresistivity beyond the RPA as a function of temperature shows no peak at all, and this is in stark contrast to the peaked behavior of the transresistivity within the RPA. The disappearance of the large high-temperature plasmon peak results from the strong increase of the drag resistivity at low temperatures: we ascribe this to the fact that the contribution to drag, made by large-angle interlayer scattering processes, becomes dominant when the many-body XC corrections are included.

As shown recently in Ref. 9, the large-angle scattering processes with $q \simeq 2 k_{F}$ play an important role in the Coulomb drag in low-density bilayers. In such samples with sufficiently small interlayer separations, $k_{F} \Lambda$ is so small that the usual exponential cutoff $\exp (-q \Lambda)$ of the interlayer $e$ - $e$ interaction is no longer effective in suppressing the large-angle scattering processes. Thus, according to previous predictions, ${ }^{40}$ the large-angle interlayer scattering events, due to the divergence of the scattering phase space near $q \simeq 2 k_{F}$, lead to a $T^{2} \ln T$ behavior of the drag resistivity at low temperatures, in lieu of the usual $T^{2}$ temperature dependence, which is driven by small-angle scattering processes. To distinguish clearly the contributions to the drag made by the 


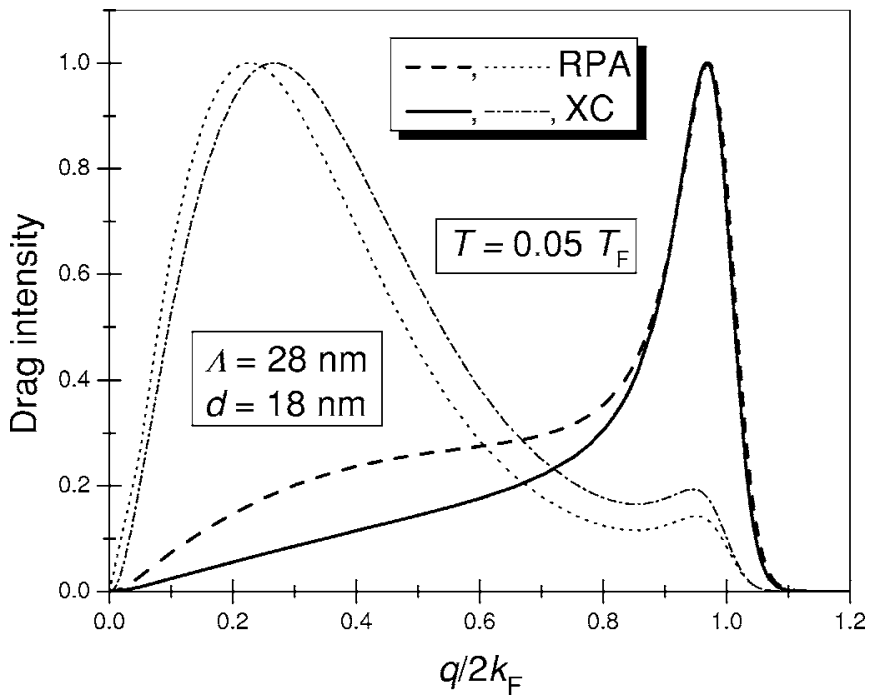

FIG. 12. Drag intensity $I(x)$ as a function of transferred momentum $x=q / 2 k_{F}$ for a low-density $n=3.8 \times 10^{10} \mathrm{~cm}^{-2}$ bilayer. For comparison the drag intensity is plotted also for a high-density $n$ $=1.5 \times 10^{11} \mathrm{~cm}^{-2}$ bilayer. The dashed (dotted) and solid (dashdotted) curves represent, respectively, the results of calculations within and beyond the RPA at $T=0.05 T_{F}$ for $n=3.8 \times 10^{10} \mathrm{~cm}^{-2}$ $\left(n=1.5 \times 10^{11} \mathrm{~cm}^{-2}\right)$. The respective values of the drag resistivity are given by the area under these curves. The curves are normalized to their particular peak values $\rho_{p}^{\mathrm{RPA}}=0.57(0.06)$ and $\rho_{p}^{\mathrm{XC}}$ $=2.71(0.01) \Omega$ for $n=3.8 \times 10^{10} \mathrm{~cm}^{-2}\left(n=1.5 \times 10^{11} \mathrm{~cm}^{-2}\right)$.

small- and large-angle interlayer scattering processes, in Fig. 12 we have plotted the dimensionless drag intensity $I(x)$ within and beyond the RPA versus the average transferred momentum $x=q / 2 k_{F}$ at low temperature $T=0.05 T_{F}$. We have normalized the drag intensity to its corresponding peak values $\rho_{p}$, so that the transresistivity $\rho_{D}=\rho_{p} \int I(x) d x$. It is evident that the $\mathrm{XC}$ effects at low density reduce essentially the component from the small-angle scattering processes so that the large-angle scattering contribution dominates the drag. Thus, the drag rate is strongly enhanced at low temperatures owing to the logarithmic corrections provided by the largeangle scattering events. By contrast, in the RPA the contribution of the small-angle scattering processes remains dominant down to the lowest temperatures (see Fig. 12).

Lack of experimental measurements of the Coulomb drag at high temperatures does not allow for the time being an experimental verification of our predictions on the position and strength of the plasmon peak in low-density bilayers. Notice, however, that at low temperatures, as shown in Fig. 13 , our numerical results are in good agreement with the experimental findings by Kellogg et al. ${ }^{9}$ for $n=3.8$ $\times 10^{10} \mathrm{~cm}^{-2}$. In this figure we have shown the drag resistivity due to exchange of particle-hole excitations. Inclusion of the dynamical part will only slightly increase the transresistivity since at such low temperatures plasmons only start to contribute. As seen in Fig. 13 the RPA calculations near $T$ $=5 \mathrm{~K}$ underestimate the experimental results by more than three times. Meanwhile, after inclusion of the XC corrections, the drag resistivity is about $88 \%$ of the experimental result. This is very good agreement against the background of the usual discrepancy between theory and experiment in determining the magnitude of the drag.

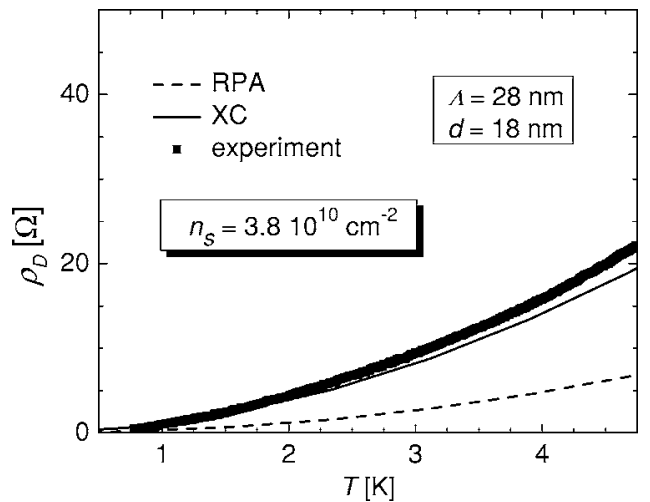

FIG. 13. The drag resistivity versus temperature for $n=3.8$ $\times 10^{10} \mathrm{~cm}^{-2}$. The solid curve represents calculations when the intraand interlayer many-body corrections are included, the dashed curve is the RPA results. The symbols are the experimental findings from Ref. 9.

In Fig. 14 we plot the drag resistivity versus temperature for six different densities. From the comparison with Fig. 1 from the experiment in Ref. 9 it is seen that our results reproduce very well all the experimental findings for the densities $n=(5.2,4.7$, and 3.8$) \times 10^{10} \mathrm{~cm}^{-2}$. For lower densities $n=(1.7,2.3$, and 3.1$) \times 10^{10}$ our calculations start to deviate from the experimental results. This is more clearly seen in Fig. 15 where we show a log-log plot of the drag resistivity versus density $n$ for three different temperatures: $T=1,2$, and $4 \mathrm{~K}$. The symbols are our calculations while straight solid line represents $n^{-4}$ dependence. This figure corresponds to Fig. 2 from Ref. 9 where it has been found that the experimental results can be very well approximated by the quartic density dependence. As seen in Fig. 15, except for very low densities, our calculations of the drag resistivity, which include the XC corrections, are also approximated by the quartic density dependence quite well. This contrasts with the RPA-based results where the drag resistivity shows $n^{-3}$ dependence.

\section{SUMMARY AND CONCLUSION}

In this paper we have investigated the role of the manybody XC effect on the dispersion of collective excitations

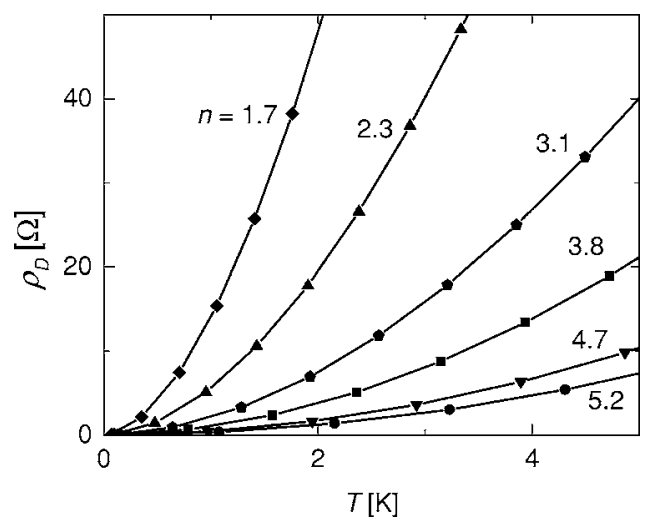

FIG. 14. The drag resistivity vs temperature for six different densities corresponding to Fig. 1 from Ref. 9. The densities are in units of $10^{10} \mathrm{~cm}^{-2}$. 


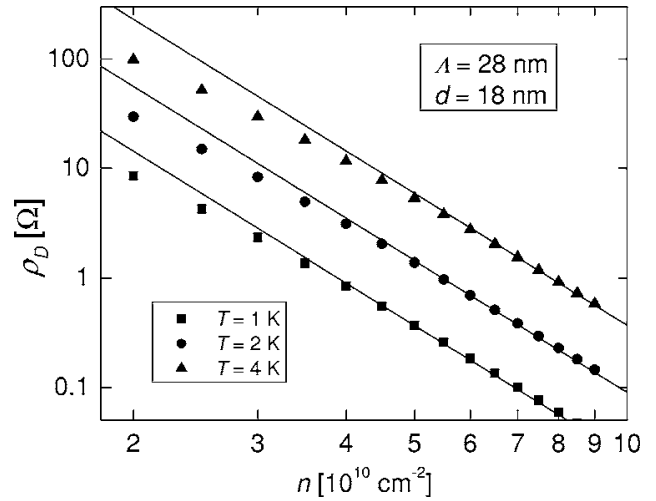

FIG. 15. Log-log plot of the transresistivity vs density for three different temperatures $T=1,2$, and $4 \mathrm{~K}$. This figure corresponds to Fig. 2 from Ref. 9. The solid lines are proportional to $n^{-4}$.

and on the frictional Coulomb drag in low-density bilayer 2DESs. We have calculated the spectrum of the bilayer plasmons and the temperature dependence of the drag resistivity in a broad range of temperatures. Our calculations include both intra- and interlayer XC effects. These effects are critical in low-density bilayers since the interlayer separation in these structures is comparable with the interparticle spacing in an individual layer. We have proposed a different approach and employed the full dynamic XC kernels of the bilayer 2DES in calculations of the acoustical and optical plasmon dispersions as well as in the drag, mediated by exchange of bilayer plasmons. In studying the many-body effects on the particle-hole contribution to drag we have still used the static exchange-controlled LFFs. The combined effect of the dynamic and static many-body XC on drag has been investigated in comparison with the RPA-based calculations of the transresistivity vs temperature for different values of the electron density. At low temperatures we have compared our results with the experimental findings from Ref. 9.

We have observed that, after the inclusion of the full dynamic XC kernels, a decrease of the electron density induces shifts of the plasmon branches in opposite directions. And this is in stark contrast to the tendency obtained within the RPA that both optical and acoustical plasmons move away from the boundary of the PHC with a decrease of the electron density. In the presence of the XC effects, the optical mode still moves away from the PHC while the acoustical branch moves closer to the PHC. The effect is so strong that at the total $r_{s}=3$ the acoustical branch enters the PHC and becomes completely destroyed at finite temperatures. These changes of the plasmon spectrum, induced by the intra- and interlayer dynamic $\mathrm{XC}$, are reflected in the temperature dependence of the plasmon-mediated drag.

After inclusion of the XC effects the upturn temperature, which is determined by the thermal excitations of the acoustical modes, exhibits a redshift, while the plasmon enhancement peak, which is mainly due to the thermal excitation of high-energy optical modes, undergoes to a blueshift with decreasing carrier concentration. Already for the total $r_{s}=2$ the upturn temperature decreases so strongly that the bilayer plasmons heavily contribute to the drag starting at temperatures below $0.1 T_{\mathrm{F}}$, which is approximately a factor of 2 smaller than the upturn temperature calculated within the RPA. The effect becomes even stronger for the total $r_{s}=3$ so that the upturn temperature disappears practically. Our results show that at low temperatures the dynamic XC effects enhance the plasmon-mediated drag while at high temperatures they have the opposite effect. We ascribe this to the enhancement of the damping of high-energy plasmons, caused by dynamic many-body XC effects.

The combined effect of the dynamic and the static manybody XC in low-density bilayers results in both quantitative and qualitative changes in the behavior of the drag resistivity. At temperatures near $0.5 T_{F}$ the transresistivity with the $\mathrm{XC}$ effects included is larger approximately by a factor of 3 than that obtained within the RPA for the in-layer $r_{s}^{\text {in layer }}=2 \sqrt{2}$. The enhancement increases strongly at low temperatures where the drag is dominated by large-angle scattering processes with $q \simeq 2 k_{F}$. We have found that the plasmon enhancement peak, which is clearly seen in the RPA-based calculations, disappears at large $r_{s}$ after the full XC corrections have been introduced. The disappearance of the large hightemperature plasmon peak is the result of the strong enhancement of the particle-hole contribution to drag at low temperatures and of the moderate suppression of the plasmonmediated drag at high temperatures when, respectively, the full static and dynamic XC corrections are included. We have ascribed this behavior to the change in the specific contributions to drag made by small- and large-angle scattering events. Our calculations demonstrate clearly that the XC effects increase the large-angle scattering component of the drag, making it the dominant contribution. This component strongly enhances the drag for two reasons. First, at low temperatures the $e$-e scattering phase space diverges near $x$ $=q / 2 k_{F} \simeq 1$. On the other hand, when $x \simeq 1$, the static intralayer LFF $G_{11}(x)$ becomes close to unity, leading to a reduction of the effective intralayer interaction $V_{\text {eff,11 }}(x)$. This by itself weakens the dynamic intralayer screening and enhances the drag. Thus, against the background of the large transresistivity at small $T$, the plasmon-mediated contribution to drag does not result in a peaked behavior of the scaled transresistivity and the total drag rate decreases monotonically in $T$. In the RPA, the drag still contains a strong smallangle scattering component which suppresses partially the enhancement at small $T$ and the plasmon peak remains visible.

Currently, the available experimental data for the lowdensity electron samples are restricted to low temperatures where the plasmon contribution to drag is small. An experimental verification of the plasmon peak disappearance requires new drag measurements in low-density electron bilayers in a broad range of temperatures close to $T_{F}$.

At low temperatures $T \ll T_{F}$ our numerical results are in good agreement with the experimental findings by Kellogg et al. ${ }^{9}$ At the upper end of the temperature interval for which data are available our calculations of the drag resistivity give about $90 \%$ of the measured drag. This agreement is especially striking in view of the usual discrepancy between theory and experiment in determination of the absolute value of the drag. Our calculations show that the drag resistivity as a function of density exhibits a $n^{-4}$ dependence for not too low densities. This is in contrast to the RPA-based calcula- 
tions of the drag resistivity and in agreement with the experimental data.

In spite of these successes one must not lose sight of the many reasonable but uncontrolled approximations that underlie this work. First of all, the very first equation for the drag resistivity, Eq. (1), is based on a decoupling approximation which factors the four-point force-force response function into a convolution product of two two-point response functions. After that, we have relied on an approximate expression for the effective interaction $W_{12}(q, \omega)$ in terms of certain local field factors which, at present, are imperfectly known to say the least. We have tried to get around the second difficulty by introducing the concept of the "reference monolayer" and by using the best available approxima- tions for its static and dynamic LFFs (see the discussion in Sec. III). In future work it will be important to understand to what extent the simple formula (1) is applicable in the strong-coupling regime and with what effective interaction $W_{12}(q, \omega)$. Also, the local field factors will have to be calculated for the bilayer system. The story is still unfolding.

\section{ACKNOWLEDGMENTS}

We thank Zhixin Qian for useful discussions and for kindly making the results of his calculations accessible prior to publication. This work was supported by KOSEF Grant No. R05-2003-000-11432-0 and NSF Grant No. DMR0313681 .
*Electronic address: smbadalyan@ysu.am

${ }^{1}$ S. M. Girvin and A. H. Macdonald, in Perspectives in Quantum Hall Effects, edited by S. Das Sarma and A. Pinczuk (Wiley, New York, 1997); J. P. Eisenstein, ibid.

${ }^{2}$ J. P. Eisenstein and A. H. MacDonald, Nature (London) 432, 691 (2004).

${ }^{3}$ M. B. Pogrebinskii, Sov. Phys. Semicond. 11, 372 (1977).

${ }^{4}$ P. J. Price, Physica B \& C 117\&118, 750 (1983).

${ }^{5}$ A. Rojo, J. Phys.: Condens. Matter 11, R31 (1999).

${ }^{6}$ G. F. Giuliani and G. Vignale, Quantum Theory of the Electron Liquid (Cambridge University Press, Cambridge, U.K., 2005), Chap. 10.

${ }^{7}$ R. Pillarisetty, Hwayong Noh, D. C. Tsui, E. P. De Poortere, E. Tutuc, and M. Shayegan, Phys. Rev. Lett. 89, 016805 (2002).

${ }^{8}$ R. Pillarisetty, Hwayong Noh, E. Tutuc, E. P. De Poortere, K. Lai, D. C. Tsui, and M. Shayegan, Phys. Rev. B 71, 115307 (2005).

${ }^{9}$ M. Kellogg, J. P. Eisenstein, L. N. Pfeiffer, and K. W. West, Solid State Commun. 123, 515 (2002).

${ }^{10}$ A.-P. Jauho and H. Smith, Phys. Rev. B 47, 4420 (1993).

${ }^{11}$ T. J. Gramila, J. P. Eisenstein, A. H. MacDonald, L. N. Pfeiffer, and K. W. West, Phys. Rev. B 47, 12957 (1993).

${ }^{12}$ H. Rubel, E. H. Linfield, D. A. Ritchie, K. M. Brown, M. Pepper, and G. A. C. Jones, Semicond. Sci. Technol. 10, 1229 (1995).

${ }^{13}$ H. Noh, S. Zelakiewicz, T. J. Gramila, L. N. Pfeiffer, and K. W. West, Phys. Rev. B 59, 13114 (1999).

${ }^{14}$ C. Jörger, S. J. Cheng, W. Dietsche, R. Gerhardts, P. Specht, K. Eberl, and K. von Klitzing, Physica E (Amsterdam) 6, 598, 2000.

${ }^{15}$ H. C. Tso, P. Vasilopoulos, and F. M. Peeters, Phys. Rev. Lett. 68, 2516 (1992).

${ }^{16}$ M. C. Bønsager, K. Flensberg, Ben Yu-Kuang Hu, and A. H. MacDonald, Phys. Rev. B 57, 7085 (1998).

${ }^{17}$ S. M. Badalyan and U. Rössler, Phys. Rev. B 59, 5643 (1999).

${ }^{18}$ U. Sivan, P. M. Solomon, and H. Shtrikman, Phys. Rev. Lett. 68, 1196 (1992).

${ }^{19}$ N. P. R. Hill, J. T. Nicholls, E. H. Linfield, M. Pepper, D. A. Ritchie, G. A. C. Jones, Ben Yu-Kuang Hu, and Karsten Flensberg, Phys. Rev. Lett. 78, 2204 (1997);

${ }^{20}$ H. Noh, S. Zelakiewicz, X. G. Feng, T. J. Gramila, L. N. Pfeiffer, and K. W. West, Phys. Rev. B 58, 12621 (1998).

${ }^{21}$ H. C. Tso, P. Vasilopoulos, and F. M. Peeters, Phys. Rev. Lett. 70, 2146 (1993).

${ }^{22}$ K. Flensberg and Ben Yu-Kuang Hu, Phys. Rev. Lett. 73, 3572 (1994); Phys. Rev. B 52, 14796 (1995).

${ }^{23}$ L. Świerkowski, J. Szymański, and Z. W. Gortel, Phys. Rev. Lett. 74, 3245 (1995); Phys. Rev. B 55, 2280 (1997).

${ }^{24}$ E. H. Hwang, S. Das Sarma, V. Braude, and A. Stern, Phys. Rev. Lett. 90, 086801 (2003).

${ }^{25}$ A. Yurtsever, V. Moldeveanu, and B. Tanatar, Solid State Commun. 125, 575 (2003).

${ }^{26}$ F. von Oppen, S. H. Simon, and A. Stern, Phys. Rev. Lett. 87, 106803 (2001); B. N. Narozhny, I. L. Aleiner, and A. Stern, ibid. 86, 3610 (2001).

${ }^{27}$ P. F. Maldague, Surf. Sci. 73, 296 (1978).

${ }^{28}$ L. Zheng and A. H. MacDonald, Phys. Rev. B 49, 5522 (1994).

${ }^{29}$ G. D. Mahan, Many-Particle Physics (Plenum, New York, 1990), p. 130.

${ }^{30}$ G. Vignale and K. S. Singwi, Phys. Rev. B 31, 2729 (1985); 32, 2156 (1985).

${ }^{31}$ K. I. Golden, H. Mahassen, G. J. Kalman, G. Senatore, and F. Rapisarda, Phys. Rev. E 71, 036401 (2005).

${ }^{32}$ P. Gori-Giorgi, S. Moroni, and G. B. Bachelet, Phys. Rev. B 70, 115102 (2004).

${ }^{33}$ G. Senatore, S. Moroni, and D. M. Ceperley, in Quantum Monte Carlo Methods in Physics and Chemistry, edited by M. P. Nightingale and C. J. Umrigar (Kluwer, Dordrecht, 1999).

${ }^{34}$ Z. Qian and G. Vignale, Phys. Rev. B 65, 235121 (2002).

${ }^{35}$ Z. Qian, Phys. Rev. B 72, 075115 (2005).

${ }^{36}$ Z. Qian, A. Constantinescu, and G. Vignale, Phys. Rev. Lett. 90, 066402 (2003).

${ }^{37}$ A. Holas and K. S. Singwi, Phys. Rev. B 40, 158 (1989); A. J. Glick and W. F. Long, ibid. 4, 3455 (1971).

${ }^{38}$ B. Davoudi, M. Polini, G. F. Giuliani, and M. P. Tosi, Phys. Rev. B 64, 153101 (2001); 64, 233110 (2001).

${ }^{39}$ T. J. Gramila, J. P. Eisenstein, A. H. MacDonald, L. N. Pfeiffer, and K. W. West, Phys. Rev. Lett. 66, 1216 (1991).

${ }^{40}$ C. Hodges, H. Smith, and J. W. Wilkins, Phys. Rev. B 4, 302 (1971). 